\title{
MULTIPLICITIES OF THE EIGENVALUES OF THE DISCRETE SCHRÖDINGER EQUATION IN ANY DIMENSION
}

\author{
DAN BURGHELEA AND THOMAS KAPPELER \\ (Communicated by Walter Littman)
}

\begin{abstract}
The following von Neumann-Wigner type result is proved: The set of potentials $a: \Gamma \rightarrow \mathbf{R}\left(\Gamma \subseteq \mathbf{Z}^{N}\right)$, with the property that the corresponding discrete Schrödinger equation $\Delta_{d}+a$ has multiple eigenvalues when considered with certain boundary conditions, is an algebraic set of codimension $\geq 2$ within $\mathbf{R}^{\Gamma}$.
\end{abstract}

1. Introduction. The classical theorem of von Neumann and Wigner [10] shows that, within the space $S$ of real symmetric $n \times n$ matrices $(n \geq 2)$, the ones with multiple eigenvalue(s) form a real algebraic set of codimension 2 . This implies, in particular, that the set of all real symmetric matrices with simple spectrum is pathwise connected, locally pathwise connected, and dense in $S$. Recently, Lax [7] shows that in a three-dimensional vector space of $(n \times n)$ symmetric matrices there exists at least a one-dimensional subspace of matrices with multiple eigenvalues ("crossing" of eigenvalues). These results were refined and generalized by Friedland, Robbin, and Sylvester [2].

In this paper the subset of all real symmetric matrices is considered which come from the discrete Schrödinger equation $\Delta_{d}+a$ on a given subset $\Gamma$ of $\mathbf{Z}^{N}$ and with boundary conditions to be specified later. By showing a certain transversality condition, an analogous result to the one of von Neumann and Wigner is shown: We prove that the set $Q_{B}$ of all potentials $a$ with the property that $\Delta_{d}+a$ has multiple eigenvalue(s) when considered with a certain boundary condition is an algebraic set of codimension $\geq 2$.

To be more precise, let $N$ and $n_{1}, \ldots, n_{N}$ be arbitrary positive numbers which define a subset $\Gamma$ in $\mathbf{Z}^{N}$ in the following way:

$$
\Gamma:=\left\{z=\left(z_{1}, \ldots, z_{N}\right): 1 \leq z_{i} \leq n_{i}, 1 \leq i \leq N\right\} .
$$

For an arbitrary function $u: \mathbf{Z}^{N} \rightarrow \mathbf{R}$, let us define the discrete Laplace operator $\Delta_{d}$, conveniently for our purposes, by

$$
\Delta_{d} u(z):=\sum_{|w-z|=1} u(w) \quad\left(z \in \mathbf{Z}^{N}\right),
$$

where $|\cdot|$ denotes the Euclidean distance. Let $a: \Gamma \rightarrow \mathbf{R}$ be an arbitrary function. Then we consider the following two eigenvalue problems of the Schrödinger equation.

Received by the editors September 29, 1986.

1980 Mathematics Subject Classification (1985 Revision). Primary 15A18, 39A12, 55M10.

The first author was supported by NSF. 
I. Dirichlet problem.

$$
\begin{gathered}
\Delta_{d} u(z)+a(z) u(z)=\lambda u(z) \quad(z \in \Gamma) . \\
u(z)=0 \text { for } z \in \mathbf{Z}^{N} \backslash \Gamma .
\end{gathered}
$$

II. Periodic problem.

$$
\Delta_{d} u(z)+a(z) u(z)=\lambda u(z) \quad\left(z \in \mathbf{Z}^{N}\right),
$$

where $a$ is periodically extended from $\Gamma$ to the whole of $\mathbf{Z}^{N}$.

$$
u\left(z_{1}, \ldots, z_{j}+n_{j}, \ldots, z_{N}\right)=e^{i \kappa_{j}} u\left(z_{1}, \ldots, z_{j}, \ldots, z_{N}\right)
$$

for given real numbers $\kappa_{j}(1 \leq j \leq N)$.

Let us denote by $Q_{B}$ the set of all potentials $a$ in $\mathbf{R}^{P}$ such that $\left(\Delta_{d}+a\right)$, considered with the boundary condition $B$, has at least one multiple eigenvalue, where $P=\prod_{i=1}^{N} n_{i}$ and $B$ is equal to I or II, staying for the different boundary conditions as given in I and II. For the reader's convenience, let us recall that by an algebraic set we mean the set of zeros of a finite collection of polynomials in some Euclidean space. An algebraic (real analytic) variety $K$ in $\mathbf{R}^{n}$ is locally the locus of the zeros of a finite collection of polynomials (real analytic functions).

THEOREM. $Q_{B}$ is an algebraic set of codimension $\geq 2$ in $\mathbf{R}^{P}$. In particular, $\mathbf{R}^{P} \backslash Q_{B}$ is connected.

REMARK 1. The continuous analogue of the Theorem above is proved in [4].

REMARK 2. It will follow from the proof of the Theorem that the statement is true for much more general difference operators than the discrete Schrödinger operators, and more general boundary conditions than the ones given here. In particular, the Theorem will hold if we replace the Laplacian $\Delta_{d}$ by a quite general, not necessarily elliptic, difference operator of arbitrary order $\geq 2$. Evidently the Theorem does not hold for difference operators of order zero and the Lemma in $\S 2$ fails to be true for such operators.

REMARK 3. Related papers are $[\mathbf{1 - 4}, \mathbf{7}, \mathbf{9}, \mathbf{1 0}]$.

2. Proof of the Theorem. In both cases $(B=\mathrm{I}$ or II), we can include the boundary conditions in the operator $\Delta_{d}+a$ which can then be represented by $P \times P$ matrices $S_{\mathrm{I}}(a)$ and $S_{\mathrm{II}}(a)$, respectively, where the potential $a$ in $\mathbf{R}^{P}$ acts diagonally. For $a$ in $\mathbf{R}^{P}$, we $\operatorname{define} f_{B, a}:=\operatorname{det}\left(S_{B}-\lambda \mathrm{Id}\right)\left(B=\mathrm{I}\right.$ or II). $f_{B, a}$ is a polynomial in $\lambda$ of degree $P$ with coefficients which are polynomials in $a(z)(z \in \Gamma)$. Clearly, $S_{B}(a)$ has a multiple eigenvalue iff $f_{B, a}$ has a multiple root, and this is true iff the discriminant $\mathbf{D}\left(f_{B, a}\right)$ of $f_{B, a}$ does vanish (cf. e.g. [6, p. 60]). Because $\mathbf{D}\left(f_{B, a}\right)$ is a polynomial in $a(z)(z \in \Gamma)$, it follows that $Q_{B}$ is an algebraic set. To compute the claimed dimension, let us fix $B$ and introduce the following notation: For $a$ in $\mathbf{R}^{P}$ given, we denote the eigenvalues of $S_{B}(a)$ in increasing order and with their multiplicities as follows:

$$
\lambda_{1}(a) \leq \cdots \leq \lambda_{P}(a)
$$

By definition, the $m$ th eigenvalue $\lambda_{m}(a)$ of $S_{B}(a)$ has multiplicity $\geq 2$ iff

$$
\lambda_{1}(a) \leq \cdots \leq \lambda_{m-1}(a)<\lambda_{m}(a)=\lambda_{m+1}(a) \leq \cdots .
$$


We define for $m=1, \ldots, P-1$

$$
\begin{aligned}
Q_{B}(m) & :=\left\{a \in \mathbf{R}^{P}: \lambda_{1}(a)<\cdots<\lambda_{m}(a)=\lambda_{m+1}(a) \leq \cdots\right\}, \\
T_{B}(m) & :=\left\{a \in \mathbf{R}^{P}: a \in Q_{B}(m) \text { and } \lambda_{m}(a)=0\right\}
\end{aligned}
$$

and

$$
T_{B}:=\bigcup_{1 \leq m \leq P-1} T_{B}(m) \quad \text { (disjoint union). }
$$

Then $Q_{B}=\bigcup_{1 \leq m \leq P-1} Q_{B}(m)$ (disjoint union) and $Q_{B}(m) \cong T_{B}(m) \times \mathbf{R}$ by the following map:

$$
T_{B}(m) \times \mathbf{R} \rightarrow Q_{B}(m), \quad(a, c) \rightarrow a+c 1,
$$

where 1 denotes the vector in $\mathbf{R}^{P}$ with all entries equal to 1 .

Once we have shown that $T_{B}(m)$ is a real analytic variety, we conclude that also $Q_{B}(m)$ is a real analytic variety and thus

$$
\operatorname{codim} Q_{B}=\min _{m} \operatorname{codim} Q_{B}(m) \geq \min _{m}\left(\operatorname{codim} T_{B}(m)\right)-1 .
$$

It will be shown with the Proposition below that $T_{B}$ is an algebraic set of codimension $\geq 3$. So we can conclude that $\operatorname{codim} T_{B}(m) \geq \operatorname{codim} T_{B} \geq 3$. In all, we get $\operatorname{codim} Q_{B} \geq 2$. To show that $T_{B}(m)$ is a real analytic variety, let $a^{0}$ be in $T_{B}(m)$. Following Kato [5], there exists a neighborhood $U$ of $a^{0}$ in $\mathbf{R}^{P}$ such that for all $a$ in $U$

$$
\lambda_{1}(a)<\cdots<\lambda_{m}(a) \leq \lambda_{m+1}(a) \leq \cdots .
$$

Then a potential $a$ in $U$ is an element in $T_{B}(m)$ iff

$$
\left.\frac{\partial^{j}}{\partial \lambda^{j}} \operatorname{det}\left(S_{B}(a)-\lambda \mathrm{Id}\right)\right|_{\lambda=0}=0 \quad(j=0,1) .
$$

This shows that $T_{B}(m)$ is a real analytic variety.

Let us recall the definition of the sets $T_{B}$ :

$$
T_{B}:=\left\{a \in \mathbf{R}^{P}: S_{B}(a) \text { has } 0 \text { as a multiple eigenvalue }\right\} .
$$

Then for $B=\mathrm{I}$ or II:

PROPOSITION. $T_{B}$ is an algebraic set of codim $\geq 3$ in $\mathbf{R}^{P}$.

PROOF. We restrict ourselves to prove the Proposition for the case where $N=2$, $n_{1}=n_{2}=n$, and $B=\mathrm{I}$. It will follow from the proof that the general case is shown in the same way. We write $T$ for $T_{\mathrm{I}}, S$ for $S_{\mathrm{I}}$, and $n^{2}$ for $P$.

$S=S(a)$ then has the form of a block Jacobi matrix:

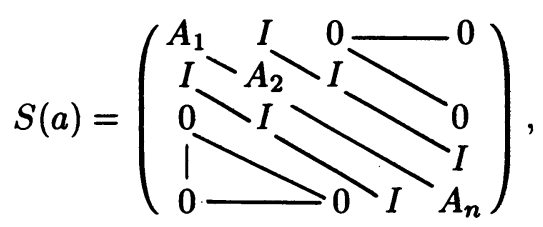


where $I$ denotes the $n \times n$ identity matrix and $A_{i}$ are $n \times n$ Jacobi matrices $(i \leq$ $i \leq n)$ given by

$$
A_{i}=\left(\begin{array}{c}
a(i, 1) \\
0
\end{array}\right.
$$

A potential $a$ in $\mathbf{R}^{n^{2}}$ is in $T$ iff $\operatorname{dim} \operatorname{Ker} S(a) \geq 2$, where Ker $S(a)$ denotes the kernel of $S(a)$. Now $\operatorname{dim} \operatorname{Ker} S(a) \geq 2$ iff all the $\left(n^{2}-1\right) \times\left(n^{2}-1\right)$ submatrices of $S(a)$ are singular. It follows that $T$ is an algebraic set in $\mathbf{R}^{n^{2}}$ and can thus be decomposed in its irreducible components $T_{i}, T=\bigcup_{i=1}^{M} T_{i}$.

It suffices to show that $\operatorname{codim}_{a} T_{i} \geq 3$ for $1 \leq i \leq M$ and any regular point $a$ in $T_{i}$ (cf. e.g. [8, p. 41]). To simplify notation let $T_{i}$ be $T_{1}$. Choose an arbitrary regular point $a^{0}$ in $T_{1}$. The idea of the proof is to express, in a neighborhood of $a^{0}$ in $T_{1}$, two coefficients out of $a_{i k}$ as functions of the others, and then to show that there is a third equation among the remaining coefficients which holds on this neighborhood and which does not hold identically on $\mathbf{R}^{n^{2}-2}$.

Let us denote by $[\alpha, i]$ the number $(\alpha-1) n+i(1 \leq \alpha, i \leq n)$. For an arbitrary $n^{2} \times n^{2}$ matrix $M$ we denote by $M((\alpha, i),(\beta, j))$ the $\left(n^{2}-1\right) \times\left(n^{2}-1\right)$ submatrix of $M$ by eliminating the $[\alpha, i]$ th row and the $[\beta, j]$ th column. Moreover, we define $b_{[\alpha, i]}:=a(\alpha, i)$.

Step 1. Let us define $F_{0}(a):=\operatorname{det} S(a)$ and $F_{k}(a):=\partial F_{k-1}(a) / \partial b_{k}\left(1 \leq k \leq n^{2}\right)$ as well as $G_{k}(a):=F_{k-1}(a)-b_{k} F_{k}(a)\left(1 \leq k \leq n^{2}\right)$. Clearly $F_{k}(a)$ and $G_{k}(a)$ are independent of $b_{1}, \ldots, b_{k}$. In particular, we have

$$
F_{k-1}(a)=b_{k} F_{k}(a)+G_{k}(a) \text { and } \operatorname{det} S(a)=b_{1} F_{1}(a)+G_{1}(a) .
$$

$F_{1}(a)$ vanishes identically on $T$. Now let us assume that $F_{2}(a), \ldots, F_{k}(a)$ are all vanishing identically in a certain neighborhood of $a^{0}$ in $T_{1}$. Due to the fact that $a^{0}$ is regular there are two possibilities:

(1) there is a neighborhood of $a^{0}$ in $T_{1}$ such that $F_{2}(a), \ldots, F_{k}(a)$ and $F_{k+1}(a)$ do vanish identically; or

(2) there exists a neighborhood of $a^{0}$ in $T_{1}$ such that $F_{k+1}$ vanishes at most on a real analytic variety of $\operatorname{codim} \geq \operatorname{codim} T_{1}+1$ contained in this neighborhood.

If the second possibility holds then one can solve the equation $0=b_{k+1} F_{k+1}(a)+$ $G_{k+1}(a)$ for $b_{k+1}$ in a neighborhood of $A^{0}$ in $T_{1}$ except on a set of points of lower dimension.

Now

$$
F_{n^{2}}(a)=\operatorname{det}\left(\begin{array}{llll}
1 & 0 & \cdots & 0 \\
0 & & & \vdots \\
\vdots & & & 0 \\
0 \ldots & \ldots & 0 & 1
\end{array}\right)=1
$$

and thus we conclude that there exists a smallest $k, 1 \leq k \leq n^{2}$, such that $b_{k}$ can be expressed as a real analytic function of $b_{k+1}, \ldots, b_{n^{2}}$ in a neighborhood $V$ of $a^{0}$ in $T_{1}$ except on a real analytic variety of lower dimension, contained in $V$.

Step 2. Let us assume that in Step 1 we could express $a(\alpha, i)$ as a real analytic function of the remaining coefficients. Then consider $0=\operatorname{det} S((\alpha, i),(\alpha, i))$. 
Applying the same procedure for $S((\alpha, i),(\alpha, i))$ as was applied for $S(a)$ in Step 1 , we conclude that there exists a neighborhood $V$ of $a^{0}$ in $T_{1}$ and $(\beta, j)$ such that $a(\beta, j)$ can be expressed as a function of $a(\gamma, k) \neq(\alpha, i)$ and $(\gamma, k) \neq(\beta, j)$ except on a set of lower dimension in $V$.

Step 3. Now let us assume that there exists a neighborhood $V$ of $a^{0}$ in $T_{1}$ and $(\alpha, i),(\beta, j)((\alpha, i) \neq(\beta, j))$ such that $a(\alpha, i)$ can be expressed as a real analytic function of the remaining coefficients of $a$, and $a(\beta, j)$ can be expressed as a real analytic function of the other coefficients different from $a(\alpha, i)$, except on a set of points of lower dimension in $V$. Then let us consider the equation

$$
0=\operatorname{det} S((\alpha, k),(\beta, j))
$$

which holds on $T$. This is a polynomial in $a(\gamma, k)$ with $(\gamma, k) \neq(\alpha, i)$ and $\neq(\beta, j)$. It thus suffices to show that $\operatorname{det} S((\alpha, i),(\beta, j))$ is not identically zero on $\mathbf{R}^{n^{2}-2}$. This will be done with the following Lemma.

LEMMA. $\operatorname{det} S((\alpha, i),(\beta, j))$ is not identically zero on $\mathbf{R}^{n^{2}-2}$.

Proof. Clearly $\operatorname{det} S((\alpha, i),(\beta, j))$ is a polynomial in $a(\gamma, k)$. We have to show that

$$
\operatorname{deg} \operatorname{det} S((\alpha, i),(\beta, j)) \geq 1 \text {. }
$$

Without loss of any generality we may and do assume that $\alpha \leq \beta$ and $i \leq j$. It then follows that $\operatorname{det} S((\alpha, i),(\beta, j))$ contains the following monomial:

$$
\left(\prod_{\substack{1 \leq k \leq n \\ 1 \leq \gamma<\alpha \text { or } \beta<\gamma \leq n}} a(\gamma, k)\right) *\left(\prod_{\substack{\alpha<\gamma \leq \beta \\ 1 \leq k \leq n \text { and } k \neq j}} a(\gamma, k)\right) *\left(\prod_{\substack{1 \leq k \leq i-1 \\ j+1 \leq k \leq n}} a(\alpha, k)\right),
$$

and thus the Lemma follows.

REMARK. If $j \leq i$, then $\operatorname{det} S((\alpha, i),(\beta, j))$ contains the following monomial:

$$
\left(\prod_{\substack{1 \leq k \leq n \\ 1 \leq \gamma<\alpha \text { or } \beta<\gamma \leq n}} a(\gamma, k)\right) *\left(\prod_{\substack{\alpha<\gamma \leq \beta \\ 1 \leq k \leq n \text { and } k \neq j}} a(\gamma, k)\right) *\left(\prod_{\substack{1 \leq k \leq j-1 \\ j+1 \leq k \leq n}} a(\alpha, k)\right) .
$$

\section{REFERENCES}

1. V. I. Arnold, Modes and quasimodes, J. Funct. Anal. 6 (1972), 94-101.

2. S. Friedland, J. W. Robbin, and J. H. Sylvester, On the crossing rule, Comm. Pure Appl. Math. 37 (1984), 19-37.

3. A. M. Micheletti, Perturbazione dello spettro dell' operatore di Laplace, in relazione ad una variazione del campo, Ann. Scuola Norm. Sup. Pisa Cl. Sci. (3) 26 (1972), 151-169.

4. Th. Kappeler, Multiplicities of the eigenvalues of the Schrödinger equation in any dimension, J. Funct. Anal. (to appear).

5. T. Kato, Perturbation theory for linear operators, 2nd ed., Grundlehren Math. Wiss. in Einzeldar., Band 132, Springer-Verlag, Berlin and New York, 1976.

6. K. Kendig, Elementary algebraic geometry, Graduate Texts in Math., vol. 44, Springer-Verlag, Berlin and New York, 1977.

7. P. D. Lax, The multiplicity of eigenvalues, Bull. Amer. Math. Soc. (N.S.) 6 (1982), 213-215.

8. R. Narasimhan, Introduction to the theory of analytic spaces, Lecture Notes in Math., vol. 25, Springer-Verlag, Berlin and New York, 1966. 
9. K. Uhlenbeck, Generic properties of eigenfunctions, Amer. J. Math. 98 (1976), 1059-1078.

10. J. von Neumann and E. Wigner, Über das Verhalten von Eigenwerten bei adiabatisdren Prozessen, Phys. Zschr. 30 (1929), 467-470.

Department of Mathematics, Ohio State University, Columbus, Ohio 43210 (Current address of Dan Burghelea)

Department of Mathematics, University of Pennsylvania, Philadelphia, PENNSYLVANIA 19104

Current address (T. Kappeler): Department of Mathematics, Brandeis University, Waltham, Massachusetts 02254 